\title{
The Hospital Readmissions Reduction Program and COPD: More Answers, More Questions
}

\author{
Valerie G Press, MD, MPH ${ }^{1 *}$, Brian J Miller, MD, MBA, MPH ${ }^{2,3}$
}

'Department of Medicine, University of Chicago Medicine, Chicago, Illinois; ${ }^{2}$ Department of Medicine, MedStar Georgetown University Hospital, Washington, DC; ${ }^{3}$ University of North Carolina Kenan-Flagler Business School, Chapel Hill, North Carolina.

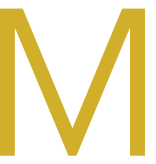

any provisions of the Affordable Care Act (ACA) have served to support the hospitalized patient. The expansion of Medicaid and the creation of state and federal insurance exchanges for the individual insurance market both significantly lessened the financial burden of hospital care for millions of Americans. Other aspects have proven more controversial, as many of the ACA's health policy interventions linked to cost and quality in new ways, implementing untested concepts derived from healthcare services research on a national scale.

The Hospital Readmissions Reduction Program (HRRP) was no exception. Based on early research examining readmissions, ${ }^{1}$ the ACA included a mandate for the Centers for Medicare and Medicaid Services (CMS) to establish the HRRP. Beginning in Fiscal Year 2013, the HRRP reduced payments for excessive, 30-day, risk-standardized readmissions covering six conditions and procedures. As the third leading cause of 30-day readmissions, chronic obstructive pulmonary disease (COPD) was included in the list of designated HRRP conditions.

This inclusion of COPD in HRRP was not without controversy; analysis of Medicare data from before the ACA's implementation demonstrated that only half of all readmissions for acute exacerbations of COPD were respiratory-related and only a third were directly related to COPD. ${ }^{2}$ Unsurprisingly, the high proportion of readmissions due to non-COPD-related causes is considered to be one of the leading factors for the failure of COPD readmission reduction programs to find significant reductions in readmissions. ${ }^{3}$ In this month's issue of the Journal of Hospital Medicine, Buhr and colleagues explore differential readmission diagnoses following acute exacerbations of COPD using a validated, national, all-payer database. ${ }^{4}$

Like many analyses of payer datasets, this study has several limitations. First, although a large area of the US was included, the data did not include all US states. Further, as the study used multiple cross-sectional data using pooling techniques, it was not truly a longitudinal study. It was additionally limited to 10 months out of the calendar year, missing December and January, which have a high seasonal prevalence of viral respiratory illness. Finally,

${ }^{\star}$ Corresponding Author: Valerie G. Press, MD, MPH; E-mail: vpress@bsd. uchicago.edu; Telephone: 773-702-5170; Twitter: @vgpress13

Published online first February 19, 2020.

Received: November 1, 2019; Revised: November 14, 2019;

Accepted: November 17, 2019

๑) 2020 Society of Hospital Medicine DOI 10.12788/jhm.3362 due to the nature of the data, COPD diagnoses were identified through administrative data known to be highly unreliable for fully capturing admissions for acute exacerbation of COPD.

Despite these limitations, the analysis by Buhr and colleagues provides additional value. They found an overall readmission rate of $17 \%$, with just under half $(7.69 \%)$ due to recurrent COPD. Patients with COPD-related readmissions were younger, had a higher proportion with Medicaid as the payer, were more frequently discharged home without services, had a shorter length of stay, and had fewer comorbidities.

Most critically, Buhr and colleagues - with a multipayer database-confirmed what researchers found in uni-payer ${ }^{5}$ and site-specific ${ }^{6}$ datasets: over half of readmissions are due to diagnoses other than COPD or respiratory-related causes. Patients readmitted due to other, unrelated diagnoses had a higher mean Elixhauser Comorbidity Index score along with higher rates of congestive heart failure and renal failure. To the practicing hospitalist, this finding supports what our internal clinical voice tells us: sicker patients are readmitted more often and more frequently with conditions unrelated to their index admission diagnosis.

The reaffirmation of the finding that the majority of readmissions are due to nonrespiratory-related causes suggests that perhaps we have a different problem than physicians and policymakers originally thought when adding COPD to the HRRP. Many COPD patients suffer from a polychronic disease, requiring a more holistic approach rather than a traditional, disease-driven, siloed approach focused solely on improving COPD-related care. It may also be true that for other subpopulations of patients with COPD, additional in-hospital and transition of care interventions are required to address patients' multimorbidity and social determinants of health.

As physicians on the front lines of the readmitted patient, hospitalists are uniquely situated to see the challenges of populations with increasing disease complexity and disease combinations. ${ }^{7}$ The HRRP policy remains controversial. This is due in large part to recent work suggesting that while the HRRP may have helped reduce readmissions, its implementation may have driven the unintended consequence of increased mortality. ${ }^{8}$ Thus, our profession faces an existential challenge to traditional care delivery models targeting diseases. What has not been well parsed by the hospital industry or policymakers is what to do about it.

Readmission of the multimorbid patient, coupled with the challenges of the HRRP, focuses our attention on the need to transition care delivery to a model that is better suited to our 
patients' needs: mass-customized, mass-produced service delivery. As physicians, we know that care delivery must be oriented around patients who have many diseases and unique life circumstances. It is our profession's greatest challenge to collaborate with researchers and administrators to help do this with scale.

\section{Acknowledgments}

The authors thank Mary Akel for her assistance with manuscript submission.

Disclosures: Dr. Press reports consulting for Vizient outside the submitted work. Dr. Miller reports consulting for the Federal Trade Commission and serving as a member of the CMS Medicare Evidence Development Coverage Advisory Committee.

Funding: Dr. Press reports funding from an NIH NHLBI R03.

\section{References}

1. Jencks SF, Williams MV, Coleman EA. Rehospitalization among patients in the Medicare Fee-for-Service Program. N Engl J Med. 2009;360(14):14181428. https://doi.org/10.1056/NEJMsa0803563.

2. Shah T, Churpek MM, Coca Perraillon M, Konetzka RT. Understanding why patients with COPD get readmitted: a large national study to delineate the Medicare population for the readmissions penalty expansion. Chest. 2015;147(5):1219-1226. https://doi.org/10.1378/chest.14-2181.

3. Press VG, Au DH, Bourbeau J, Dransfield MT, Gershon AS, Krishnan JA, et al. An American thoracic society workshop report: reducing COPD hospital readmissions. Ann Am Thorac Soc. 2019;16(2):161-170. https://doi. org/10.1513/AnnalsATS.201811-755WS.

4. Buhr R, Jackson N, Kominski G, Ong M, Mangione C. Factors associated with differential readmission diagnoses following acute exacerbations of COPD. J Hosp Med. 2020;15(4):219-227. https://doi.org/10.12788/jhm.3367.

5. Sharif R, Parekh TM, Pierson KS, Kuo Y-F, Sharma G. Predictors of early readmission among patients 40 to 64 years of age hospitalized for chronic obstructive pulmonary disease. Annals ATS. 2014;11(5):685-694. https://doi. org/10.1513/AnnalsATS.201310-3580C.

6. Glaser JB, El-Haddad H. Exploring novel Medicare readmission risk variables in chronic obstructive pulmonary disease patients at high risk of readmission within 30 days of hospital discharge. Ann Am Thorac Soc. 2015;12(9):12881293. https://doi.org/10.1513/AnnalsATS.201504-228OC

7. Sorace J, Wong HH, Worrall C, Kelman J, Saneinejad S, MaCurdy T. The complexity of disease combinations in the Medicare population. Popul Health Manag. 2011;14(4):161-166. https://doi.org/10.1089/pop.2010.0044

8. Wadhera RK, Joynt Maddox KE, Wasfy JH, Haneuse S, Shen C, Yeh RW. Association of the hospital readmissions reduction program with mortality among medicare beneficiaries hospitalized for heart failure, acute myocardial infarction, and pneumonia. JAMA. 2018;320(24):2542-2552. https://doi. org/10.1001/jama.2018.19232. 